\title{
Immunohistochemical detection of Langerhans cells in dental granulomas and radicular cysts
}

\author{
Luciano Cincurá Santos • Eduardo Antônio Gonçalves Ramos • \\ Clarissa Araújo Silva Gurgel · Edmar José Borges de Santana • \\ Jean Nunes dos Santos
}

Received: 25 January 2007 / Accepted: 22 March 2007/Published online: 11 April 2007

(C) Springer Science+Business Media B.V. 2007

\begin{abstract}
Objective Dental granulomas (DGs) and radicular cysts (RCs) are chronic periapical lesions frequently involving the jaws. Langerhans cells (LCs) are dendritic cells responsible for the presentation of antigens to $\mathrm{T}$ lymphocytes. This study examined the expression of LCs in DG and RCs by immunohistochemical staining.

Study Design Eighteen cases of DGs and 26 cases of RCs were analyzed using anti-CD1a marker.

Results CD1a-labeled LCs were observed in $11.1 \%$ of DGs and in $69.2 \%$ of RCs, showing a significant correlation $(P<0.0001$; Fisher's test). In DGs, LCs were only observed in granulation tissue, showing discrete immunostaining density. In RCs, LCs exhibited both a round and a dendritic shape in all epithelial layers. Although a correlation was observed between immunostaining density and epithelial thickness, as well as between immunostaining and inflammatory intensity, the differences were not significant in radicular cysts.
\end{abstract}

L. C. Santos

School of Dentistry, Federal University of Bahia, Salvador, Bahia, Brazil

E. A. G. Ramos - C. A. S. Gurgel

Laboratory of Histopathology, Gonçalo Moniz Research Center,

Oswaldo Cruz Foundation, Salvador, Bahia, Brazil

E. J. B. de Santana

Laboratory of Oral Pathology, School of Dentistry, Federal

University of Bahia, Salvador, Bahia, Brazil

J. N. dos Santos $(\bowtie)$

Laboratory of Oral Surgical Pathology, School of Dentistry, Federal University of Bahia, Avenida Araujo Pinho, 62, Canela, Salvador, Bahia Cep: 40110-15, Brazil

e-mail: jeanunes@ufba.br
Conclusion Langerhans cells provide important insight into the immunopathogenesis of chronic periapical lesions.

Keywords Radicular cyst - Dental granuloma . Immunohistochemistry $\cdot$ Langerhans cell

\section{Introduction}

Bacterial endotoxins, as well as cytokines originating from the immunoinflammatory process seem to influence the development of dental granulomas (DG) and radicular cysts (RC). After pulp necrosis, the dissemination and installation of bacteria in the periapex stimulate and activate innate and acquired defense mechanisms, with vascular and cellular events leading to the development of DGs and RCs (Meghji et al. 1996; Marton and Kiss 2000). According to the literature, an RC may arise from a preexisting DG or by induction of Malassez epithelial rests. This cystic formation appears as epithelial elements proliferate, with the consequent occurrence of a cavity filled with liquid or semiliquid material, events that are related to immunoinflammatory processes (Meghji et al. 1996; Marton and Kiss 2000; Piattelli et al. 2002). In addition, some morphological features found in these lesions such as cholesterol crystals, foamy macrophages and foreign-body type material, among others, seem to induce or potentiate this inflammatory event (Sjogren et al. 2002; Yamazaki et al. 2004).

Langerhans cells (LC) are professional antigen-presenting cells derived from the bone marrow, which are responsible for the presentation of antigens to $\mathrm{T}$ lymphocytes. These cells have also been reported to promote the differentiation of plasma cells from B lymphocytes. Furthermore, dendritic cells direct $\mathrm{T}$ helper lymphocytes in the 
definition of a Th1 or Th2 immune response (Holíková et al. 2001)

LC have been related to some dermatological, oncological, viral and oral diseases such as leukoplakia, gingivitis, actinic cheilitis and, more recently, odontogenic cysts (Reynolds et al. 1995; Santos 2000; Sequier et al. 2000; Piatelli et al. 2002). The goal of the present study was to analyze the presence of LC in DGs and RCs by immunohistochemistry using the CD1a antibody, in order to better understand the etiopathogenesis of these chronic periapical processes.

\section{Methods}

After Institutional Ethics Committee approval, 18 cases of DGs and 26 cases of RCs selected from the files of the Surgical Oral Pathology Laboratory, School of Dentistry of the Federal University of Bahia (FOUFBA). All cases were evaluated and selected based on the anatomopathological reports.

For morphological analysis, material fixed in formalin and embedded in paraffin was cut into $4-\mu \mathrm{m}$ thick sections. The hematoxylin/eosin-stained slides of each case were submitted to a new histological exam by light microscopy in order to describe the main morphological aspects representative of each lesion. The inflammatory infiltrate was classified into discrete, moderate or intense according to the method adapted from Tsai et al. (2004). Epithelial thickness was defined as atrophic (2-10 cell layers) or hyperplastic (>10 cell layers) according to Moreira et al. (2000).

Immunohistochemical stains were performed on formalin-fixed and paraffin-embedded $3 \mu \mathrm{m}$ thick sections. The tissue sections were deparaffined and a monoclonal antibody against CD1a (Clone NCL-CD1a, Novocastra Laboratories) was applied with EnVision HRP System (DAKO Corporation, Carpinteria, USA). For antigen retrieval, conditions included sections boiled in EDTA, $\mathrm{pH}$ 8.0 , at a constant temperature of $97^{\circ} \mathrm{C}$. Endogenous peroxidase was blocked with peroxidase block solution provided in the EnVision kit, for $10 \mathrm{~min}$ and the slides were washed with Tris-buffered saline.

The tissues sections were then exposed to antibody CD1a at 1:30 diluition for $30 \mathrm{~min}$ at room temperature, using a antibody diluent with background reducing components (DAKO Corporation, Carpinteria, USA). Immunohistochemical reactions were developed with diaminobenzidine as the chromogenic peroxidase substrate and the slides were counterstained with Harris hematoxylin. Human tonsil tissue was used as a positive control to validate the results of immunostains. Negative controls included replacement of the primary antibody with nonimmune bovine serum.
The density (quantity) and location of these positively labeled cells were analyzed by two experienced observers by light microscopy at $200 \times$ and $400 \times$ magnification in the most representative fields of each specimen. The CD1a-positive cells were defined as dendritic when they assumed a more stellate morphology. And when they had very short cytoplasmatic extensions, the CD1a-positive cells were defined as round-shaped.

After immunohistochemistry, the immunoreactive cells were analyzed semiquantitatively taking into account the following parameters: presence or absence of immunostaining (positive, negative), location (epithelium: basal, middle and superficial layer, capsule and granulation tissue), and labeling density. The immunostaining density was established by the determination of the number of CD1a-positive cells in representative fields of all specimens. Specimens showing the largest and smallest number of CD1a-positive cells were used as reference and classified as presenting intense (+++) and discrete (+) immunostaining, respectively. The other specimens were classified as presenting moderate $(++)$ immunostaining (adapted from Albuquerque et al. 2003).

The likelihood ratio and Fisher's tests were used for statistical analysis. A $P$ value $<0.05$ was considered to be significant.

\section{Results}

A total of 44 periapical lesions were studied. Clinical data obtained from patient records indicated that there were 24 men and 19 women with ages ranging from 04 years to 69 years. There was no information considering the gender in one case.

CD1a-labeled cells were identified as a brown color irrespective of staining intensity. CD1a-labeled cells were observed in $45,5 \%(n=20)$ of the lesions studied. CD1alabeled LC were detected in $11,1 \% \quad(n=2)$ of DGs exhibiting discret immunostaining density and in $69,2 \%$ $(n=18)$ of RCs with a significant association between immunostaining and the type of lesion $(P<0,0001$, Fisher's test). The immunostaining densities in the cyst lining epithelium varied as following: discrete $(+)$ density in eigh cases, moderate $(++)$ in four cases and intense $(+++)$ in seven cases. They were observed in all epithelial layers but predominated in the middle layer $(29.6 \%)$, followed by the basal layer (25.9\%). LC were also detected in granulation tissue and capsules in the RCs (Fig. 1).

Positive CD1a cells showed a dendritic morphology mainly on the surface and the middle region of the epithelium, whereas the round morphology predominated in the basal region. However, dendritic forms were also observed in the suprabasal zone and the round form in the 
Fig. 1 Immunohistochemical staining of Langerhans cells in chronic periapical lesions. Dental granuloma: (A) Langerhans cell discretely labeled for CD1a present among plasma cells (approximately 400×). Radicular cyst: (B) Discrete immunostaining of a round Langerhans cell amidst epithelial cells (approximately 400×); (C) Moderate immunostaining of Langerhans cells present in the middle layer showing few dendritic prolongations (approximately 200×) (D) Intense immunostaining of Langerhans cells present in different epithelial layers; (E) Dendritic and round Langerhans cells in the middle layer of the hyperplastic epithelial lining (approximately 100×); (F)

Round Langerhans cells in the suprabasal layer of the epithelium (approximately $100 \times)$
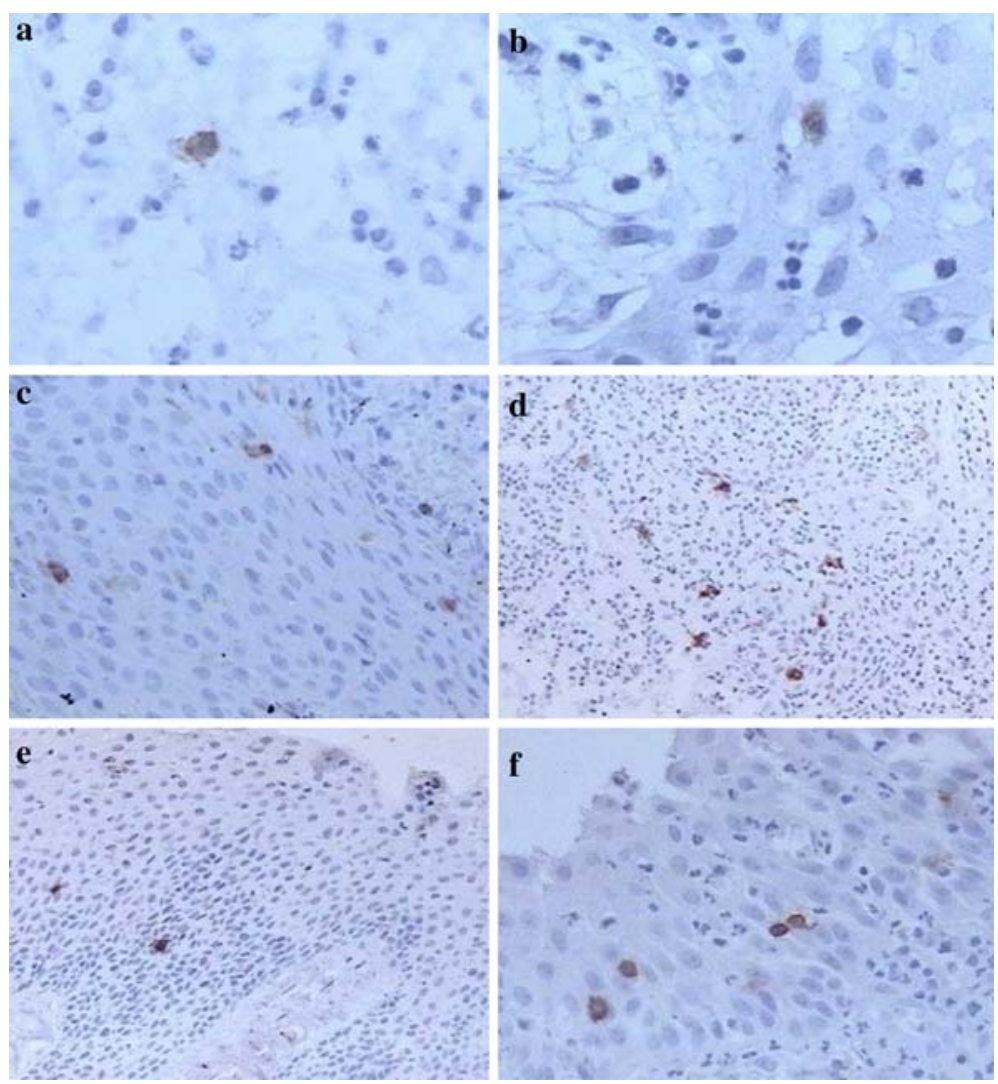

middle region. Half of the 18 cystic CD1a-positive lesions presented an atrophic epithelium and the other half a hyperplastic epithelium. However, no significant correlation was observed between immunostaining density and epithelial thickness $(P=0.513$, likelihood ratio; Table 1). Among the 20 CD1a-positive periapical lesions studied, $50 \%$ presented an intense chronic inflammatory infiltrate. However, no significant correlation was observed between inflammatory intensity and immunostaining density $(P=0$. 473, likelihood ratio; Table 2).

\section{Discussion}

Many studies have used S-100 as a marker for LC, but other surface markers can be used for the detection of these cells, such as HLA-DR, an antigen-presenting molecule, CD29, a cell adhesion molecule, and CD54. However, LC express high levels of CD1a, langerin and E-cadherin, specific markers for these cells which are not found in other types of dendritic cells. (Lombardi et al. 1993; Peña-Cruz et al. 2003; Sequier et al. 2003). Some authors reported the use of fascin as a marker of LC but it also detects interdigitating cells, follicular cells and veiled cells (Sugihara et al. 2005).

LCs were detected in $69.2 \%$ of the RCs studied and in $11.1 \%$ of the DGs, showing a significant correlation between immunostaining and the type of lesion $(P=0.0001$, Fisher's test). All cases of DGs exhibited discrete immunostaining, whereas variable densities of CD1a-labeled LC were observed in RCs. However, immunostaining was not significant in the cases of DGs, in contrast to the results reported by Suzuki et al. (2001). This result might be

Table 1 Distribution of immunostaining according to epithelial thickness of the radicular cyst

\begin{tabular}{lllll}
\hline Thickness & Density & & & \\
\cline { 2 - 5 } & Discrete $(\%)$ & Moderate $(\%)$ & Intense $(\%)$ & $4(57.1)$ \\
\hline Atrophic & $4(57.1)$ & $1(25.0)$ & $3(42.9)$ & $9(50.0)$ \\
Hyperplastic & $3(42.9)$ & $3(75.0)$ & $7(38.9)$ & $9(50.0)$ \\
Total & $7(38.9)$ & $4(22.2)$ & $(100.0)$ \\
\hline
\end{tabular}

$P=0.513(>0.05$, likelihood ratio) 
Table 2 Distribution of immunostaining according to the intensity of the inflammatory infiltrate

\begin{tabular}{|c|c|c|c|c|}
\hline \multirow[t]{2}{*}{ Infiltrate } & \multicolumn{4}{|l|}{ Density } \\
\hline & Discrete $(\%)$ & Moderate $(\%)$ & Intense $(\%)$ & Total $(\%)$ \\
\hline Discrete & $3(33.3)$ & $0(0.0)$ & $1(14.3)$ & $4(20.0)$ \\
\hline Moderate & $2(22.2)$ & $1(25.0)$ & $3(42.9)$ & $6(30.0)$ \\
\hline Intense & $4(44.4)$ & $3(75.0)$ & $3(42.9)$ & $10(50.0)$ \\
\hline Total & $9(45.0)$ & $4(20.0)$ & $7(35.0)$ & $20(100.0)$ \\
\hline
\end{tabular}

$P=0.473(>0.05$, likelihood ratio)

attributed to absence of epitheliated granulomas in our sample.

Like Piattelli et al. (2002) we did not detect LC in all cases of RCs, in contrast to Suzuki et al (2001) who observed immunostaining for these cells in $100 \%$ and $95 \%$ of this type of lesion, respectively. This smaller number of CD1a-labeled cells might be related to the process of cell repair, which is characterized by a phase of quiescence or regression of cystic growth probably as a result of conventional endodontic treatment (Suzuki et al. 2001).

Another hypothesis for the decrease in LC number in some RCs has been proposed by Babi (1998), who attributed the reduction in the number of LC to a process of cell apoptosis after antigen presentation to CD4+ $\mathrm{T}$ cells. In addition, variations in the methods used and in immunohistochemical sensitivity may interfere with the results. Sequier et al. (2000) suggested that this decrease in LC number may also be related to the migration of these cells to regional lymph nodes after their activation by contact with external pathogens.

According to Cutler and Jotwani (2004), LCs are present in the basal and suprabasal layers in the cystic epithelium and may be concentrated in distinct regions of this epithelium. In addition, the morphology of LC varies according to their degree of maturation. Mature LCs are characterized by an irregular stellate shape and long and thin cytoplasmic prolongations and are generally found in the suprabasal layers of the epithelium. In contrast, immature cells show a more regular round shape, few or no dendritic prolongations and are preferentially located in the basal layer of the epithelium (Moreira et al. 2000; Cutler and Jotwani 2004). Similar to the findings of Akhlaghi and Dourov (1995), in the present study these dendritic forms were not only detected in the basal layer but also scattered throughout the epithelium. In addition, we also observed round LC in the middle and superficial layers of the epithelium. This finding might be explained by the hypothesis of Sequier et al. (2000) who suggested a structural modification of these cells at the time of capture and antigen presentation to $\mathrm{T}$ lymphocytes, leading to a reduced immunological stimulus and a greater specialization in antigen presentation.
Although we observed a higher density of LC in lesions with an intense $(50 \%)$ and moderate $(30 \%)$ inflammatory infiltrate, as also reported by Pringle et al. (1992), Suzuki et al. (2001), Albuquerque et al. (2003), statistical analysis did not reveal a significant correlation between inflammatory intensity and immunostaining density $(P=0.473$, likelihood ratio). This finding was similar to those found by Piattelli et al. (2002). We believe that the inflammatory intensity is not only related to an increased amount of antigen in the lesion as reported by Alburquerque et al. (2003) but also to an exacerbated response of the defense mechanisms of the individual.

Some investigators have correlated epithelial thickness with the inflammatory intensity of the lesion (Moreira et al. 2000; Suzuki et al. 2001). According to these authors, the inflammatory stimulus provided mainly by growth factors and cytokines triggers epithelial proliferation. Suzuki et al. (2001) observed a significant direct correlation between the density of CD1a-labeled LC and the proliferative potential of the epithelium in $\mathrm{RCs}$, residual radicular cysts and epitheliated granulomas. In the present study, the relationship between positive immunostaining and hyperplastic epithelium was observed in $50 \%$ of cases of RCs, although this finding was not statistically significant. This result might be explained by a possible involution of the lesions studied in cases when conventional endodontic treatment had been performed.

A significant association was observed between positive CD1a staining and the type of lesion. In DGs, CD1alabeled LC were observed in granulation tissue, whereas in RCs labeled cells were detected in all cystic epithelium, as well as in granulation tissue and in the capsule. In the case of DGs, immunostaining density was always discrete. In contrast, discrete and intense immunostaining densities predominated in $\mathrm{RCs}$, with no significant correlation being observed between immunostaining density and epithelial thickness or inflammatory intensity.

In conclusion, Langerhans cells provide important insight into the immunopathogenesis of chronic periapical lesions as DGs and RCs, possibly in response to pathogens coming from root canal. In addition, studies of new cell receptors, such as Toll-like receptors, responsible for the 
control and activation of dendritic cells (Barton and Medzhitov 2002) should be conducted in an attempt to better understand the response of chronic periapical lesions to other direct regulators of the immune response.

Acknowledgments We thank Ana Carvalho, Mírian de Jesus Moraes, Maria de Lourdes dos Santos and Nancy Silva Santos for excellent technical assistance. This work was supported by FAPESB, Grants 1431040047950 and 200/04.

\section{References}

Akhlaghi N, Dourov N (1995) Langerhans cells in odontogenic cysts. A retrospective study based on 142 cases. Bull Group Int Rech Sci Stomatol Odontol 38(3-4):71-76

Albuquerque RL Jr, Miguel RC, Costa AL et al (2003) Correlation of c-erbB-2 and S-100 expression with the malignancy grading and anatomical site in oral squamous cell carcinoma. Int J Exp Pathol 84(6):259-265

Babi LFS (1998) Las células de Langerhans en la inmunidad cutánea. Con especial referencia a la dermatitis atópica. Acta Dermatol 3:173-181

Barton G, Medzhitov R (2002) Control of adaptive immune responses by Toll-like receptors. Curr Opin Immunol 14(3):380-383

Cutler CW, Jotwani R (2004) Antigen-presentation and the role of dendritic cells in periodontitis. Periodontol 2000 35:135-57

Holíková Z, Hercogova J, Pizak J et al (2001) Dendritic cells in the skin and mucosa: what́s new. J Eur Acad Dermatol Venereol 15(2):116-120

Lombardi T, Hauser C, Budtz-Jorgensen E (1993) Langerhans cells: structure, function and role in oral pathological conditions. J Oral Pathol Med 22(5):193-202

Marton IJ, Kiss C (2000) Protective and destructive immune reactions in apical periodontitis. Oral Microbiol Immunol 15(3):139-150

Meghji S, Qureshi W, Henderson B et al (1996) The role of endotoxin and cytokines in the pathogenesis of odontogenic cysts. Arch Oral Biol 41(6):523-531

Moreira PR, Santos DF, Martins RD et al (2000) CD57+ cells in radicular cyst. Int Endod J 33(2):99-102
Peña-Cruz V, Ito S, Dascher CC et al (2003) Epidermal Langerhans cells efficiently mediate CD1a-dependent presentation of microbial lipid antigens to T cells. J Invest Dermatol 121(3):517-521

Piattelli A, Rubini C, Iezzi G et al (2002) CD1a-positive cells in odontogenic cysts. J Endod 28(4):267-268

Pringle GA, Daley TD, Veinot LA et al (1992) Langerhańs cell histiocytosis in association with periapical granulomas and cyst. Oral Surg Oral Med Oral Pathol 74(2):186-192

Reynolds NJ, Yi JY, Fisher GJ et al (1995) Down-regulation of Langerhans cell protein kinase C-beta isoenzyme expression in inflammatory and hyperplastic dermatoses. $\mathrm{Br} \mathrm{J}$ Dermatol 133(2): $157-167$

Santos JN (2000) Expressão imuno-histoquímica de citoqueratinas, CD1a, S-100, p53, p21, PCNA e Ki-67 em queilite actínica. Thesis, University of São Paulo, Brazil

Sequier S, Godeau G, Leborgne M et al (2000) Quantitative morphological analysis of Langerhans cells in health and diseased human gingival. Arch Oral Biol 45(12):1073-1081

Sequier S, Bodineau A, Godeau G et al (2003) Langerin+ versus CD1 a+ Langerhans cells in human gingival tissue: a comparative and quantitative immunohistochemical study. Arch Oral Biol 48(4):255-262

Sjogren U, Mukohyama H, Roth C et al (2002) Bone-resorbing activity from cholesterol-exposed macrophages due to enhanced expression of interleukin-1alpha. J Dent Res 81(1):11-16

Sugihara A, Okamoto H, Horio T (2005) Effects of UVB on fascin expression in dendritic cells and Langerhans cells. J Dermatol Sci 40(3):177-185

Suzuki T, Kumamoto H, Ooya K et al (2001) Immunohistochemical analysis of CD1a-labeled Langerhans cells in human dental periapical inflammatory lesions - correlation with inflammatory cells and epithelial cells. Oral Dis 7(6):336-343

Tsai CH, Weng SF, Yang LC et al (2004) Immunohistochemical localization of tissue-type plasminogen activator and type I plaminogen activator inhibitor in radicular cysts. J Oral Pathol Med 33(3):156-161

Yamazaki M, Cheng J, Hao N et al (2004) Basement membrane-type heparan sulfate proteoglycan (perlecan) and low-density lipoprotein (LDL) are co-localized in granulation tissues: a possible pathogenesis of cholesterol granulomas in jaw cysts. J Oral Pathol Med 33(3):177-184 